\title{
Physics based model for online fault detection in autonomous cryogenic loading system
}

\author{
Ali Kashani ${ }^{\mathrm{a}}$ and Ekaterina Ponizhovskaya ${ }^{\mathrm{b}}$ and Dmitry Luchinsky ${ }^{\mathrm{c}}$ and Vadim \\ Smelyanskiy ${ }^{\mathrm{d}}$ and Jared Sass ${ }^{\mathrm{e}}$ and Barbara Brown ${ }^{\mathrm{e}}$ and Anna Patterson-Hine ${ }^{\mathrm{d}}$
}

\author{
${ }^{a} S G T$, Inc., Greenbelt, $M D$, USA \\ ${ }^{b} S G T$, Inc., Greenbelt, MD, USA \\ ${ }^{c}$ Mission Critical Technologies, Inc., El Segundo, CA, USA \\ ${ }^{d}$ Ames Research Center, NASA, Moffett Field, CA, USA \\ ${ }^{e}$ Kennedy Space Center, NASA, Moffett Field, CA, USA
}

\begin{abstract}
We report the progress in the development of the chilldown model for rapid cryogenic loading system developed at KSC. The nontrivial characteristic feature of the analyzed chilldown regime is its active control by dump valves. The two-phase flow model of the chilldown is approximated as one-dimensional homogeneous fluid flow with no slip condition for the interphase velocity. The model is built using commercial SINDA/FLUINT software. The results of numerical predictions are in good agreement with the experimental time traces. The obtained results pave the way to the application of the SINDA/FLUINT model as a verification tool for the design and algorithm development required for autonomous loading operation.
\end{abstract}

Keywords: Cryogenic loading, two-phase flow, homogeneous flow, SINDA/FLUINT.

PACS: 07.20.Mc; 47.11.-j; 47.60.-i.

\section{INTRODUCTION}

A rapid cryogenic propellant loading system is currently being developed at KSC. It is envisaged that this system will be employed in the remote and autonomous launch operations. The latter can be defined as the capability to accomplish launch vehicle propellant load and drain without human interaction. In practice, such ability depends on many factors including e.g. the propellant load and drain protocols, availability of automatic and remotely operable hardware etc. Importantly, the system and subsystems should be able to change their behavior in response to unanticipated events. The complexity of this problem dictates the necessity of development of a physics based model for loading operation that can reproduce accurately the time traces of pressure and temperature during the loading, predict system response to various deviations from the loading protocol, detect and localize system faults online.

In this paper we report a progress in development of a SINDA/FLUINT model of cryogenic fluid loading with the focus on modeling the chilldown regime, which is controlled by the control valves. The commercial SINDA/FLUINT software is widely used for an analysis of heat flow in aerospace applications. This model will be used to verify the design and the algorithms of the fault detection, isolation, and mitigation in autonomous cryogenic loading systems.

The paper is organized as follows. In the next section the description of the system is provided. The two-phase model and of the approximations used in SINDA/FLUINT are discussed in Section III. The results of the simulations are compared with the experimental time traces in the Section IV. Finally, the conclusions are down and the future work is discussed in the Conclusions. 


\section{CRYOGENIC LOADING SYSTEM}

The geometry of the model of the cryogenic loading system developed at KSC for testing autonomous regimes of operation is shown in the FIGURE 1. The system consists of the storage tank (left) and external tank (right) connected by a pipeline. The total length of the line is about $45 \mathrm{~m}$. The characteristic speed of sound in the gas phase is about $200 \mathrm{~m} / \mathrm{sec}$. The diameter of the stainless pipe varies along the pipeline from 6 inch to 1 inch. The thickness of the walls is approximately $3 \mathrm{~mm}$. In the earlier tests analyzed in the current research, the pipe walls were exposed to radiation with corresponding heat flux estimated as $\approx 1000 \mathrm{~kW} / \mathrm{m}^{3}$.

The transfer of the cryogenic fluid from the storage tank to the external tank is driven by high pressure in the storage tank. Pressure of the external tank remains approximately one atmosphere during transfer. The transfer consists of the three main stages: (i) chilldown, (ii) fast fill, and (iii) replenish. In this work we primarily focus on the chilldown process as the most nontrivial part of the cryogenic transfer operation.

The chilldown takes approximately 25 minutes and uses up about $180 \mathrm{Gal}$ of liquid nitrogen. The chilldown is initiated when the main valve next to the storage tank is opened. The flow through the system remains blocked for another $\approx 100 \mathrm{sec}$ because the valve 4 at the middle of the pipe line remains closed. Once valve 4 is opened the gas and liquid gas mixture begin to flow through the pipes cooling the facility. Most of the liquid nitrogen is used to fill in the pipes. About $10 \%$ of this volume is spent to cool the pipes, about $15 \%$ of it is used to compensate for the pipes heating due to radiation.

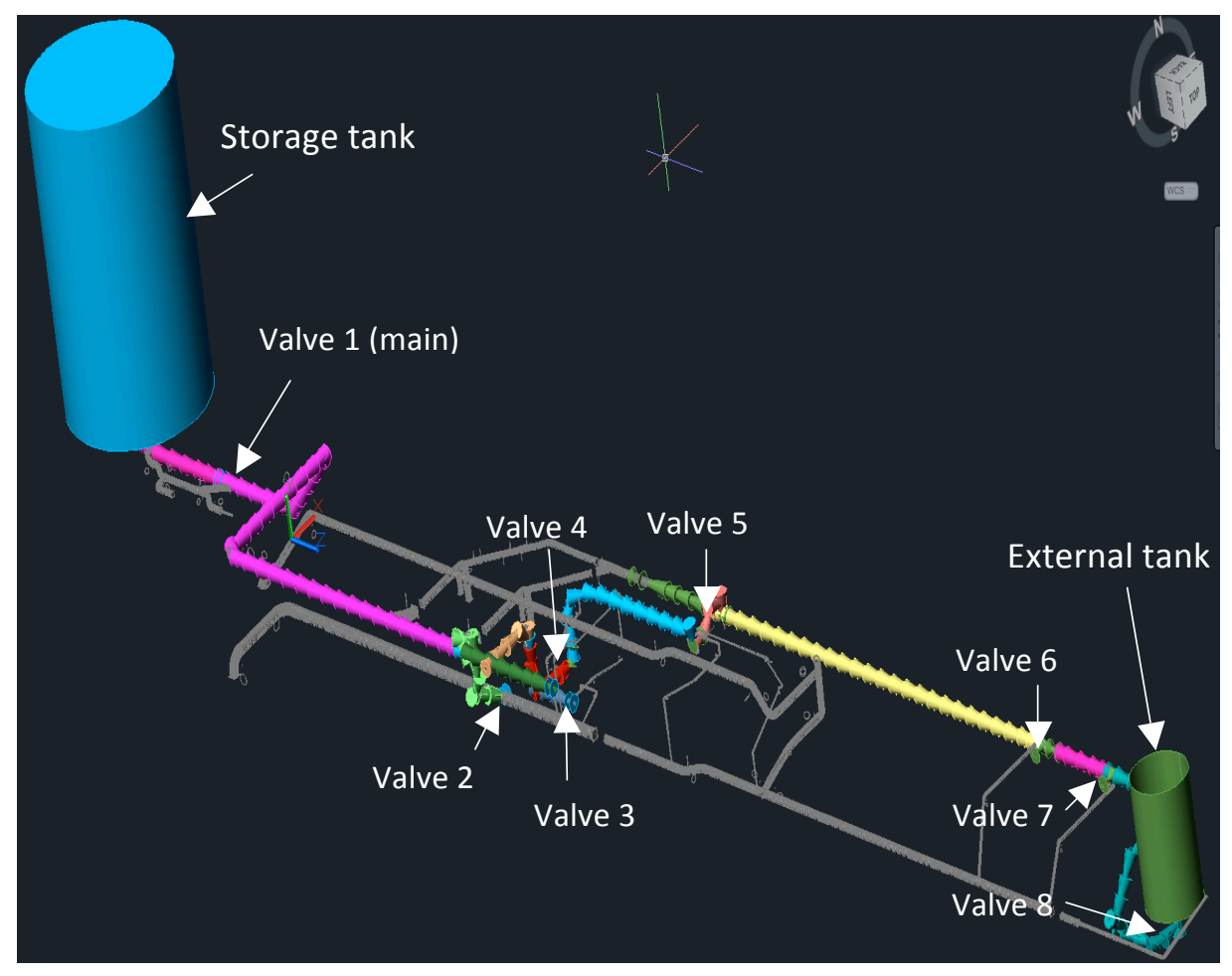

FIGURE 2. Geometry of the model of the cryogenic loading system built in ThermalDesktop.

The important feature of the experimental testbed developed at KSC is the existence of relatively large number of remotely operated control valves. These valves are used not only to reduce or block the flow along the pipe line but also to dump a given amount of the nitrogen gas or liquid practically at any location along the pipe line. These valves are used to actively control chilldown of the system. The location of some of the control valves is shown in the FIGURE 3.

Another interesting feature of the experimental setup is intentional strong radiative heating of the pipes that leads to multiple experimentally observed boiling crisis during chilldown and strong phase separation during fast fill and replenish. The dynamics of local boiling crisis and phase separation is controlled mainly by opening and closing the dump valves. By the end of the chilldown the whole facility should reach the liquid nitrogen temperature and all the dump valves should normally be closed. 
An example of the temperature variation along the pipeline during chilldown is shown in the FIGURE 2. Different lines in this figure correspond to the different sensors location. The left line corresponds to the location near storage tank. The right line corresponds to the location near external tank. Initially all the sensors indicate ambient temperature. During the chilldown the temperature gradient is created in the system. The cold front propagates from the storage tank to the external tank (see FIGURE 4) following the propagation of the liquid nitrogen front.

Different slopes of the temperature variations measured by the sensors indicate different flow quality at a given location. The lower the quality of the flow the steeper is the slope. The steepest slope appears in the FIGURE 2 almost as an abrupt temperature drop, which corresponds to the violent evaporation of liquid nitrogen in the contact with the pipe walls. Evaporating nitrogen is mixing with local gas quickly reducing its temperature. By the end of the chilldown process, the liquid front approaches the external tank as indicated by the temperature drop in the most right temperature sensor.

The presence of the boiling crisis can be seen in the FIGURE 2 (see e.g. red line) as a rapid increase of the local temperature due to the wall dry out promoted by the radiative heating. The subsequent temperature drop is achieved by opening of the downstream dump valve.

Several techniques can be used to model the nontrivial thermodynamics of the chilldown. From the point of view of online fault detection isolation and recovery it is desirable to develop the simplest possible model that capture correctly all the required phenomena. In this manuscript we report the results of an analysis of one of such models the homogeneous non-steady model of chilldown developed using commercial software SINDA-FLUINT.

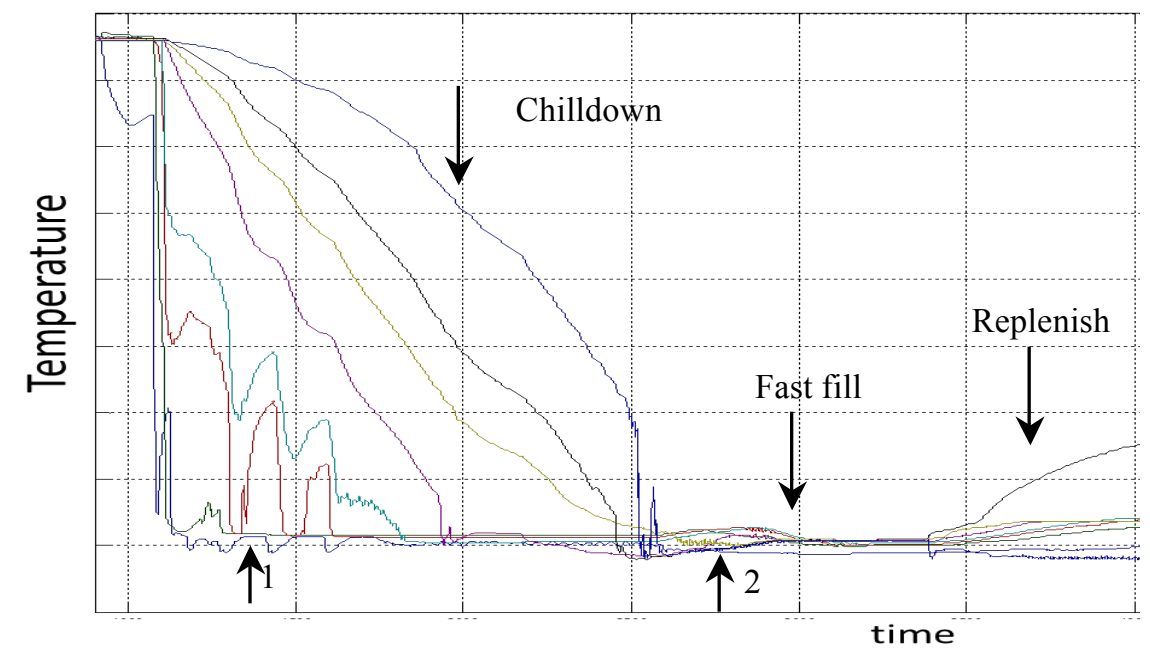

FIGURE 2. Temperature variation along the pipeline. 1 indicates boiling crisis. 2 phase separation

\section{SINDA/FLUINT MODEL OF THE CRYOGENIC SYSTEM}

The two-phase flow is modeled as a one-dimensional homogeneous flow with no slip condition. The governing equations can be written in the form conservation laws for the mass, momentum, and energy of the mixture (see e.g. [1])

$$
\begin{aligned}
& A \frac{\partial \rho}{\partial t}+\frac{\partial A \rho u}{\partial x}=0, \\
& A \frac{\partial \rho u}{\partial t}+\frac{\partial A \rho u^{2}}{\partial x}=-A \frac{\partial p}{\partial x}-\left(\tau_{w} l_{w}\right)_{2 \phi}-\rho A g \sin \theta, \\
& A \frac{\partial \rho E}{\partial t}+\frac{\partial A \rho u H}{\partial x}=q_{w} l_{w},
\end{aligned}
$$

where $\rho$ is the density, $u$ is the velocity, and $p$ is the pressure of the flow with total energy $E=c_{v} T+u^{2} / 2$ and total enthalpy $H=E+p / \rho$. The no-slip condition $u=u_{g}=u_{l}$ (subscripts $g$ and $l$ indicate gas and liquid 
respectively) was assumed in derivation of eq. (1). The cross-sectional area of the pipe is $A, l_{w}$ is the perimeter of the wall, $\theta$ is the angle of pipe axis with horizontal line, and $x$ is the length along the pipe.

The mixture properties are defined as follows

$$
\rho=\alpha_{g} \rho_{g}+\alpha_{l} \rho_{l}, \quad e=\chi_{g} e_{g}+\chi_{l} e_{l}, \quad h=\chi_{g} h_{g}+\chi_{l} h_{l},
$$

where $\alpha_{g(l)}$ is the void fraction of gas (liquid), $\chi_{g(l)}$ is the corresponding mass fraction, $e_{g(l)}$ and $h_{g(l)}$ are specific energy and enthalpy of gas (liquid).

\section{SINDA/FLUINT implementation}

The two-phase frictional losses $\left(\tau_{w} l_{w}\right)_{2 \phi}$ are determined using default flow regime based correlations [2] in SINDA/FLUINT, which recognizes four simplified regimes: bubbly, slug, annular, and stratified. The default Chen's boiling correlations (see e.g. [3]) are used to determine heat flux $q_{w}$.

The flow is thermally coupled to the pipe wall, which temperature $T_{w}$ is described by the following equation

$$
c_{w} \rho_{w} d_{w} \frac{\partial T_{w}}{\partial t}=-q_{w}+q_{e x}
$$

The system of equations (1) and (2) is modelled in SINDA/FLUINT as follows. The conservation equations for the mass and energy are solved by dividing the pipeline into a number of control volumes, which are called lumps. There are total around 100 lumps in the present model. The lumps are connected by paths, which solve for the momentum conservation equations on a staggered grid. The heat transfer to the wall is modeled by introducing ties between the lumps and the wall control volumes. The external radiation is modeled as a constant heat flux to the pipe wall.

\section{Control valves}

As was mentioned in the introduction the chilldown in the experimental cryogenic system was actively controlled using the dump valves. In addition, the flow through the system was blocked during first $100 \mathrm{sec}$ of the chilldown by the valve 4 (see FIGURE 5). As a result the dynamics of the chilldown is largely determined by the dynamics of the valve operation.

In the present model, the valves are introduced as local variable losses. The time dependence of these losses follows explicitly the dynamics of the valve operation in the experimental system by including the corresponding tables into the model.
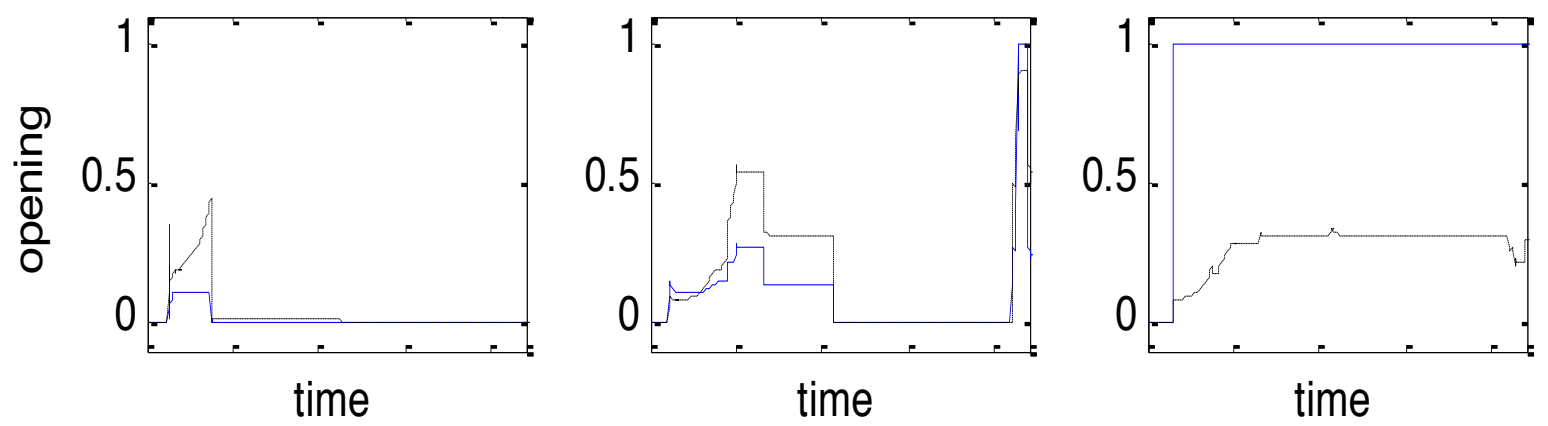

FIGURE 3. The dynamics of the valve 2 (left), valve 3 (middle), and vale 4(right) shown by blue solid lines in comparison with the flow rate $\mathrm{(au})$ through these valves.

We will now show that even this strongly simplified can reproduce reasonably well the experimentally observed time series data

\section{COMPARISON WITH THE EXPERIMENT}

From the point of view of modeling cryogenic loading system modeling the chilldown regime render itself as a challenging task (see e.g. [4,5]). During the chilldown the rapid equilibration between three phases (heated pipe 
wall, subcooled cryogenic liquid, and superheated vapor) causes nontrivial dynamics of temperature variations. Modeling this dynamics is a long-standing problem of two-phase flow. And the ability of the algorithm to reproduce this dynamics is one the key approaches to its validation.

The comparison of the experimental time traces of the temperature variation during with numerical predictions obtained using SINDA/FLUINT model is shown in the FIGURE 4. It can be seen from the figure that the model can with good accuracy reproduce the main dynamical features of the temperature variation along the line
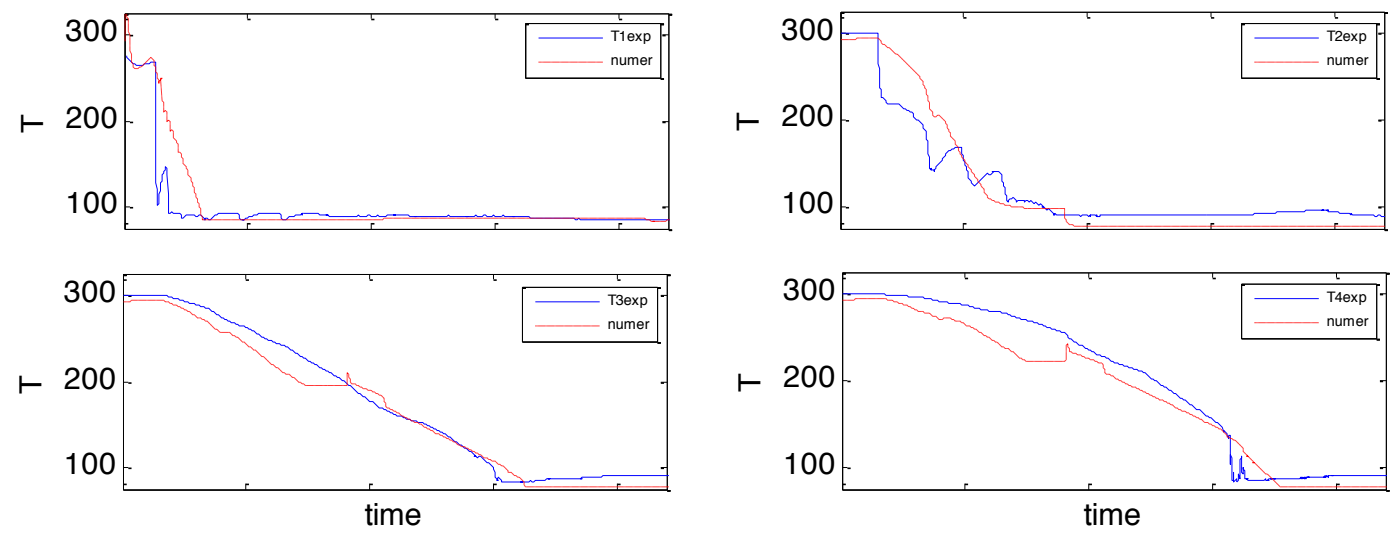

FIGURE 4. The comparison of the experimental time traces for the temperature (blue solid line) with the numerical predictions (red dashed line) for four different locations along the line: $\mathrm{T} 1$ - close to the storage tank; T2 - before valve 4; T3 - right after valve 4; T4 - closer to the end of the line.
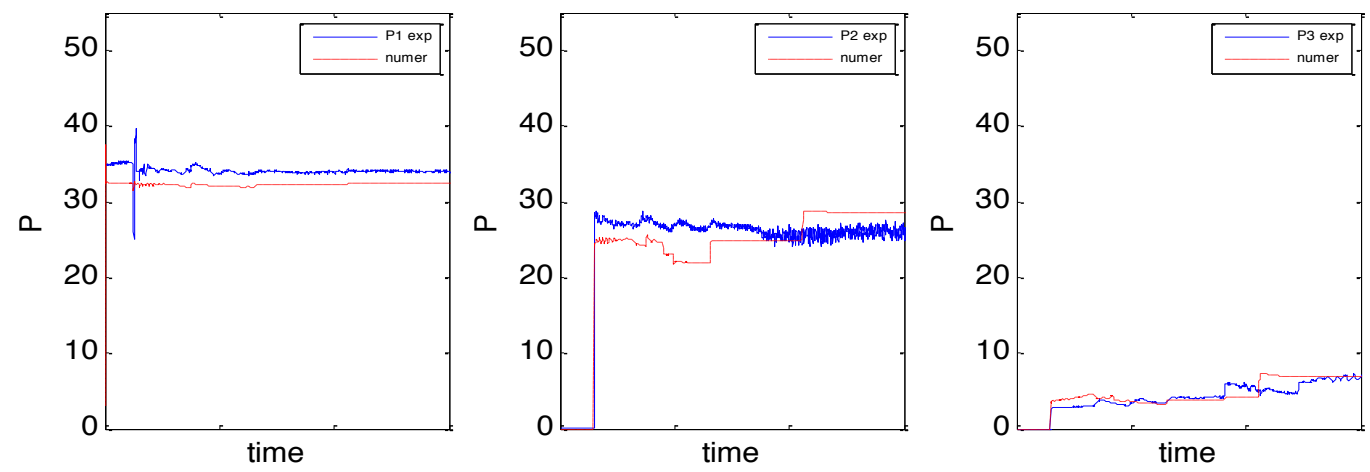

FIGURE 5. The comparison of the experimental time traces for the pressure (blue solid line) with the numerical predictions (red dashed line) for three different locations along the line: P1 - close to the storage tank; P2 - right after valve 4; P3 - closer to the end of the line.

\section{ACKNOWLEDGMENTS}




\section{REFERENCES}

1. H. Staedtke, Gasdynamic Aspects of Two-Phase Flow: Hyperbolicity, Wave Propagation Phenomena, and Related Numerical Methods, Darmstadt: John Wiley \& Sons, 2006.

2. C.J. Crowley, M.G. Izenson, Design Manual for Microgravity Two-Phase Flow and Heat Transfer, Defense Technical Information Center; Technical Report, AD-A214 937, 1989.

3. American Society of Heating, Refrigerating, and Air Conditioning Engineers. ASHRAE Handbook of Fundamentals: ASHRAE, 1981.

4. M. F. Cross, A. K. Majumdar, J. C. Bennett, and R.B. Malla, Journal of Spacecraft and Rockets 39, 284-289 (2002).

5. A.K. Majumdar and S.S.Ravindran, Journal of Propulsion and Power 27, 620-630 (2011). 